\title{
Human African trypanosomiasis in a Belgian traveller returning from the Masai Mara area, Kenya, February 2012
}

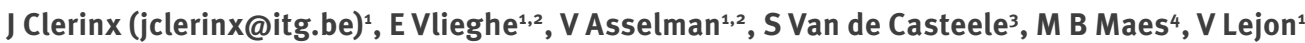

1. Department of Clinical Sciences, Institute of Tropical Medicine, Antwerp, Belgium

2. Department of Tropical Diseases, University Hospital Antwerp, Belgium

3. Department of Nephrology, Hospital St. Jan Brugge, Belgium

4. Haematology laboratory, University Hospital Antwerp, Belgium

Citation style for this article:

Clerinx J, Vlieghe E, Asselman V, Van de Casteele S, Maes MB, Lejon V. Human African trypanosomiasis in a Belgian traveller returning from the Masai Mara area, Kenya, February 2012

Euro Surveill. 2012;17(10):pii=20111. Available online: http://www.eurosurveillance.org/ViewArticle.aspx?Articleld=20111

A Belgian traveller was diagnosed with human African trypanosomiasis (HAT) due to Trypanosoma brucei rhodesiense nine days after visiting the Masai Mara area in Kenya. He presented with an inoculation chancre and was treated with suramin within four days of fever onset. Two weeks earlier, HAT was also reported in a German traveller who had visited the Masai Mara area. Because no cases have occurred in the area for over 12 years, this may indicate a focal cluster of HAT.

\section{Case report}

We report here the diagnosis of human trypanosomiasis (HAT) due to Trypanosoma brucei rhodesiense in a Belgian man who visited the Masai Mara National Reserve in Kenya from 7 to 9 February 2012. A summary of this case was reported through ProMED-mail on 22 February 2012 [1]. A similar case had been reported from Frankfurt, Germany, in a traveller who had visited the Masai Mara area in January 2012 [2], which is described further in this issue [3].

The Belgian patient stayed at a lodge at the southern end of the Mara River for one night and participated in game tracking excursions on two occasions in the Reserve. He returned to Belgium on 13 February. He presented at the St. Jan's Hospital in Bruges on 19 February with a history of high-grade fever, malaise and headache that had been present for three days. He also had a painless and discrete chancre on his arm that he had noticed only two days earlier (Figure 1). The patient was suspected to have malaria and a Giemsastained thick blood smear was prepared for microscopy. No malaria parasites were seen, but instead trypanosomes were identified on the thick smear, and subsequently confirmed on a thin smear (Figure 2). The patient was immediately transferred to the Tropical Diseases Unit at the University Hospital Antwerp for treatment. Pre-treatment blood analysis showed a high parasitemia (of more than one trypanosome per field on microscopy at 100x magnification), marked thrombocytopenia (47,000 platelets $/ \mu \mathrm{L})$, alanine aminotransferase (ALAT) and aspartate aminotransferase (ASAT) at three times the upper limit of normal, and moderately increased C-reactive protein. The trypanosome species was identified as T.b. rhodesiense by PCR detection of the serum resistance-associated gene [4].

Treatment with suramin (1g) was initiated on 20 February, after a test dose of $100 \mathrm{mg}$ was well tolerated. In our setting, suramin is given once weekly for five weeks. After 12 hours, an electrocardiography showed diffuse but transient S-T elevation often seen in acute $T$. b. rhodesiense trypanosomiasis. However, the levels of cardiac enzymes remained within normal limits and no cardiac dysfunction was seen on echocardiography. Trypanosomes were cleared from the blood 24 hours after suramin was given.

\section{FIGURE 1}

Inoculation chancre of human African trypanosomiasis in a Belgian traveller returning from the Masai Mara area, Kenya, February 2012

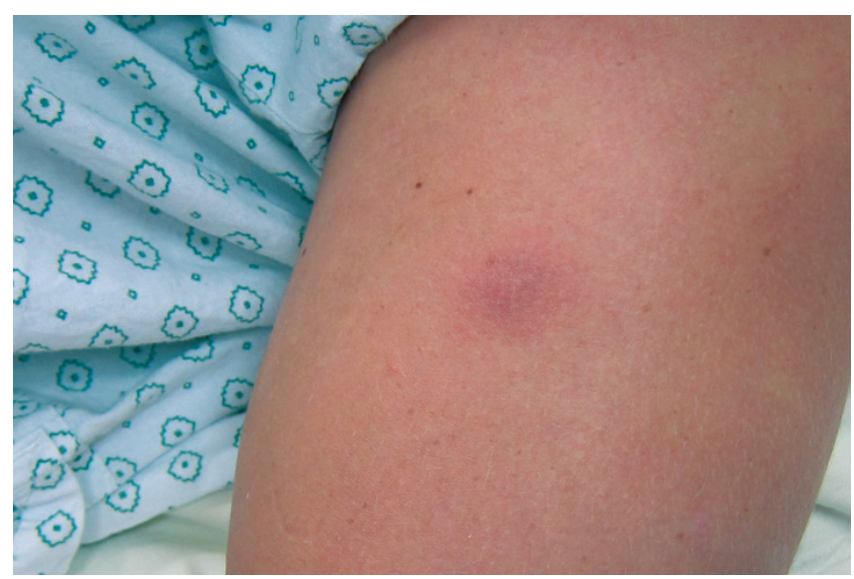


A generalised papulopruriginous rash appeared 36 hours after the start of treatment, lasting for four days. The patient became afebrile two days after treatment; the chancre persisted until the fifth day. He recovered clinically by the seventh day and a platelet count and liver function tests had returned to near normal values by then. A cerebrospinal fluid examination performed on the seventh day, when the second dose of suramin was given, showed a normal cell count and no trypanosomes.

The time lapse from presumed inoculation until the onset of fever was 11 days. Treatment was initiated on the 13th day after presumed inoculation. Such relatively early treatment should prevent invasion of the brain by the parasite [5].

The trypanosome inoculation chancre observed on admission is an important clinical sign present in about two thirds of patients [6]. As in our case, it may be discrete and easily overlooked by physicians unfamiliar with this rare disease.

\section{Background}

Trypanosoma brucei rhodesiense is endemic in East and southern Africa and is transmitted to humans and game alike by tsetse flies of the Glossina morsitans group, which feed during the day. It accounts for the majority of imported HAT cases [6]. In Kenya, HAT is rare, in Kenyans and travellers alike. Until the cases reported this year, the last two autochtonous cases reported date from 2006 and 2009 and originated from the north-west part of the country (P. Simarro, personal communication, 21 February 2012). The Masai Mara Conservation Area in south-west Kenya receives about 300,000 visitors annually. Nowadays, the area also includes adjacent farms around the original Masai
Mara National Reserve. In the last 12 years, HAT has not been seen in travellers visiting Masai Mara, in contrast to the situation in the adjacent Serengeti National Park in Tanzania [7].

Among patients presenting fever after a travel in the tropics, $T$. $b$. rhodesiense HAT remains a very rare event [8]. Parasitemia is usually elevated during the acute febrile phase. This aids diagnosis, as even for microscopists unfamiliar with the parasite, trypanosomes can be easily seen on a Giemsa-stained routine thick blood smear, and often on a thin blood smear too. Trypanosomes have an unmistakable shape (Figure 2).

\section{Discussion}

Acute $T . b$. rhodesiense HAT is a medical emergency. Multi-organ failure may occur early in the course of the febrile phase with a high mortality risk, similar to that of severe malaria. In a large series of imported T. b. rhodesiense HAT cases, the case fatality rate was $4.3 \%$, associated either with late diagnosis during the acute febrile stage or with meningo-encephalitic stage treatment toxicity [6]. In 2007, a German patient infected with $T . b$. rhodesiense HAT in the Serengeti, Tanzania, died of multi-organ failure five days after the onset of the acute febrile phase. The diagnosis had been missed by another physician in Zanzibar, where trypanosomiasis does not occur, four days before the patient died despite fever and the presence of a chancre. The patient was treated with antimalarials without a blood test having been done [9]. Patients require immediate treatment with suramin, after a test dose to observe any hypersensitivity to the drug. If suramin cannot be obtained within a day, immediate treatment with pentamidine has to be considered. Although not the first choice for $T$. $b$. rhodesiense HAT, pentamidine was effective in a few imported $T$. b. rhodesiense HAT

\section{FIGURE 2}

Trypanosomes in (A) thick and (B) thin blood smears at diagnosis from a Belgian traveller returning from the Masai Mara area, Kenya, February 2012

A

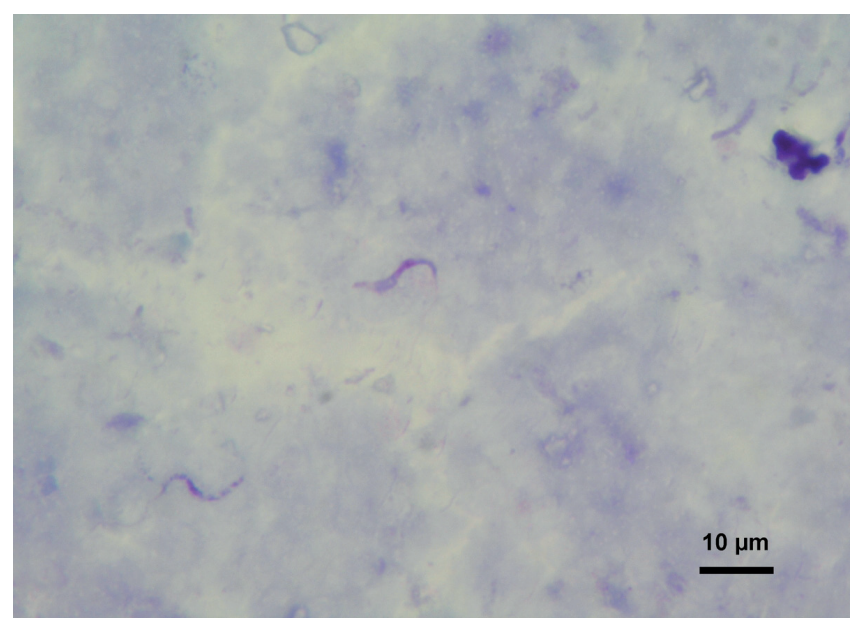

B

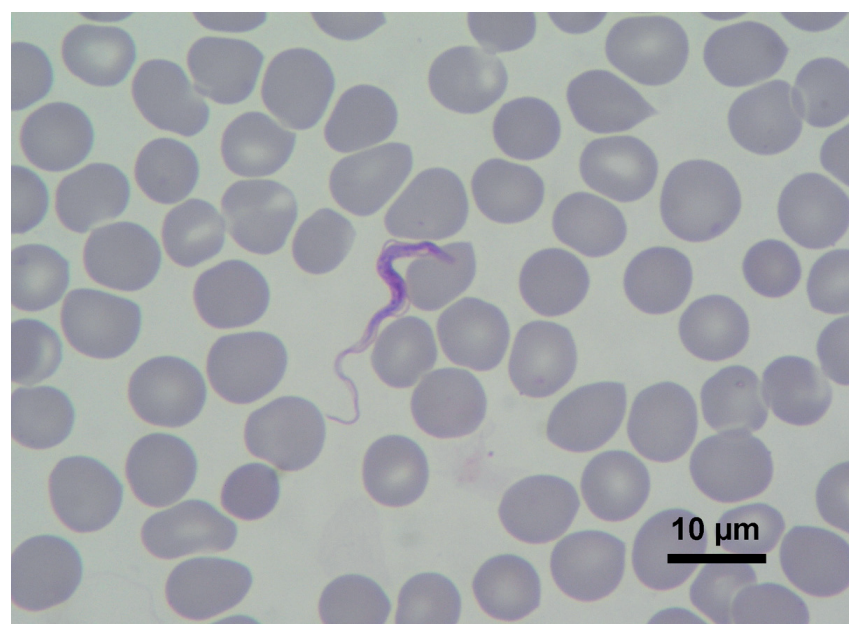


cases as the sole treatment given $[6,10,11]$. Suramin and other HAT medications can be obtained from the World Health Organization headquarters in Geneva, Switzerland, at very short notice.

Our patient had stayed overnight in a lodge close to the Mara River, in the south of the Masai Mara area, whereas the German patient had stayed in another lodge about $30 \mathrm{~km}$ further north (P. Simarro, personal communication, 21 February 2012) (Figure 3). However, both travellers may have visited the same area during one of the daytime game-watching excursions.

The coincidence of two $T . b$. rhodesiense HAT cases infected after visiting the Masai Mara area suggests a possible, incipient focal cluster, similar to the onset of an outbreak seen in travellers visiting the adjacent Serengeti in 2001 and 2002 [7]. T. b. rhodesiense HAT is endemic in both game reserves and game migrate annually between both areas. The ecosystems of the Masai Mara area and the northern part of the Serengeti are nearly identical. At any given time, only very few tsetse flies are infected with $T$. $b$. rhodesiense, which can infect humans. Most carry zoonotic species ( $T$. congolense, $T$. vivax, T. b. brucei) that do not have the serum resistance-associated gene required to resist parasite lysis after inoculation in humans.
Although $T . b$. rhodesiense HAT remains an exceptionally rare imported infectious disease, early recognition and treatment assures a favourable outcome.

\section{Acknowledgments}

The pictures of the thick and thin blood smears were kindly provided and prepared by I. Potters, Clinical Laboratory, Institute of Tropical Medicine, Antwerp.

\section{References}

1. ProMED-mail. Trypanosomiasis-Belgium ex Kenya: (Masa Mara). Archive number: 20120222.1049305. 22 Feb 2012. Available from: http://www.promedmail.org/direct. php?id=20120222.1049305

2. ProMED-mail. Trypanosomiasis-Human Germany ex Kenya: Masai Mara. Archive number: 20120202.1031130. 2 Feb 2012. Available from: http://www. promedmail.org/direct. php?id=20120202.1031130

3. Wolf T, Wichelhaus T, Göttig S, Kleine C, Brodt HR, JustNuebling G. Trypanosoma brucei rhodesiense infection in a German traveller returning from the Masai Mara area, Kenya, January 2012. Euro Surveill. 2012;17(10): pii=20114. Available from: http://www.eurosurveillance.org/ViewArticle. aspx?Articleld $=20114$

4. Radwanska M, Chamekh M, Vanhamme L, Claes F, Magez S, Magnus E, et al. The serum resistance-associated gene as a diagnostic tool for the detection of Trypanosoma brucei rhodesiense. Am J Trop Med Hyg. 2002;67(6):684-90.

\section{FIGURE 3}

Masai Mara area, Kenya, with the approximate location of the lodges where the German and Belgian travellers stayed, January-February 2012

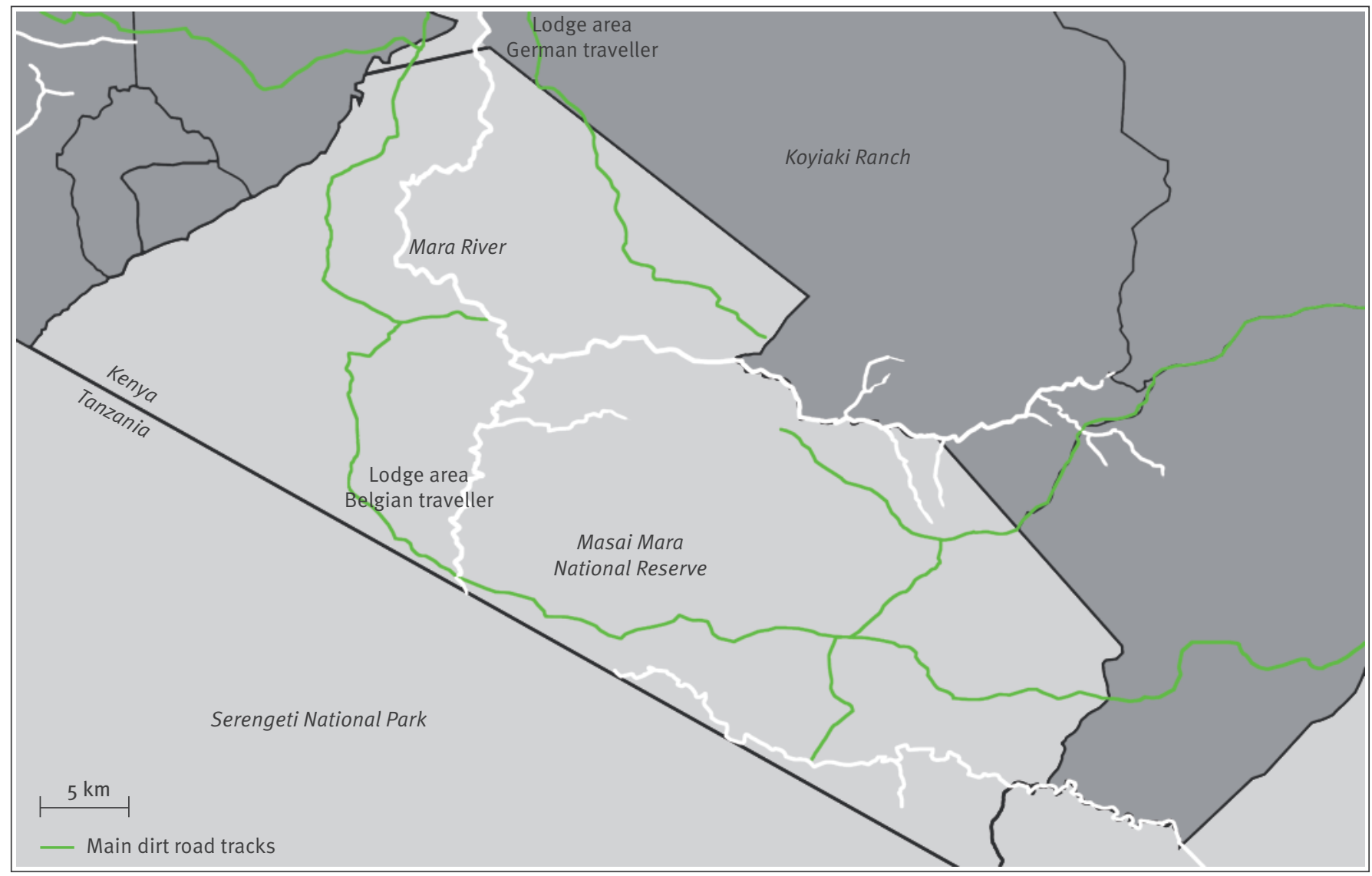


5. MacLean L, Chisi JE, Odiit M, Gibson WC, Ferris V, Picozzi K, Sternberg JM. Severity of human african trypanosomiasis in East Africa is associated with geographic location, parasite genotype, and host inflammatory cytokine response profile. Infect Immun. 2004;72(12):7040-4.

6. Simarro PP, Franco JR, Cecchi G, Paone M, Diarra A, Ruiz Postigo JA, et al. Human African trypanosomiasis in nonendemic countries (2000-2010). J Travel Med. 2012;19(1):44-53.

7. Jelinek T, Bisoffi Z, Bonazzi L, van Thiel P, Bronner U, de Frey A, et al. Cluster of African trypanosomiasis in travellers to Tanzanian national parks. Emerg Infect Dis. 2002;8(6):634-5.

8. Bottieau E, Clerinx J, Schrooten W, Van den Enden E, Wouters $R$, Van Esbroeck M, et al. Etiology and outcome of fever after a stay in the tropics. Arch Intern Med. 2006;166(15):1642-8.

9. Klaassen B, Smit YG. [A fatal outcome of acute East African sleeping sickness in Tanzania]. Tijdschr Infect. 2009;4(2):61-5. Dutch.

10. Gautret P, Clerinx J, Caumes E, Simon F, Jensenius M, Loutan $\mathrm{L}$, et al. Imported human African trypanosomiasis in Europe, 2005-2009. Euro Surveill. 2009 Sep 10;14(36):pii=19327. Available from: http://www.eurosurveillance.org/ViewArticle. aspx?Articleld $=19327$

11. ProMED-mail. Trypanosomiasis - Poland ex Uganda (Queen Elizabeth National Park). Archive number: 20090810.2844. 10 Aug 2009. Available from: http://www.promedmail.com/direct. php?id=20090810.2844 